\title{
Trans-Obturator Tape Operation Using MONARC Technique (Outside-In Technique) --- Revisited Objective and Subjective Assessment
}

\author{
${ }^{1}$ Dr Fouzia Memon, ${ }^{2}$ Mr. Mohamed Matar \\ ${ }^{I}$ (Consultant Obstetrician and Gynaecologist) Cumberland Infirmary, Newtown Road, Carlisle CA2 $7 H Y$ \\ ${ }_{2}^{2}$ Consultant Obstetrician and Gynaecologist) Cumberland Infirmary, Newtown Road, Carlisle CA2 7 HY
}

\begin{abstract}
:
Objective: This study was undertaken to describe and evaluate the outcome of trans-obturator tape (TOT) operation using Outside-In technique without cystoscopy. This procedure was carried out either solely or as a part of pelvic reconstructive surgery.

Design: Retrospective cohort study with follow up objective and subjective assessment.

Population: 84 women were presented with urodynamic stress incontinence at North Cumbria NHS Trust. They were treated with transoturator tape operation using outside-in technique between the years 2007 and 2008.

Method: Eighty-four women underwent trans-obturator tape operation (outside-in technique) without cystoscopy. Diagnosis of stress urinary incontinence (SUI) was made by uro-dynamic studies. They were all operated only by a single surgeon. The mean length of follow-up was 6 months. Operative complications were recorded. The anatomic integrity of urethra, the anterior vaginal wall was objectively assessed at follow up visit. Subjective assessment was made during patient consultation. Objective failure was defined as persistence of urodynamic stress urinary incontinence. Subjectively, failure was defined mainly as persistence of stress incontinence symptoms. Other related symptoms are noted like voiding difficulties and sexual dysfunction.

Results: In the study data eighty four women underwent a TOT operation. The mean age was 50 years (38-63). Pre-operatively, of those 84 patients, SUI was diagnosed in 62 patients while mixed urinary symptoms were present in 22 patients. Transobturator tape operation was carried out using MONARC subfacial hammock system in all women. Additional pelvic surgical procedures were performed according to the presence of other pelvic defects. No intraoperative complications were recorded. The estimated blood loss was $125 \mathrm{~mL}$ (range, 50-200 mL). None of the patient had suspected bladder or urethral injury intraoperatively. Transient urinary retention was observed in $8 \%(n=7)$ of women in immediate postoperative period. Three women developed urinary tract infection in immediate postoperative period and in one woman urethral pus was noted which resolved with the help of oral antibiotics. Postoperative objective assessment was made with speculum examination and stress provocation tests like cough and valsalva manoeuvres. There was no stress incontinence on stress provocation tests. Two women developed de novo urge incontinence that was treated with anticholinergics. Two complained of worsening urge incontinence symptoms and none of the women having concurrent urge incontinence symptoms showed any improvement in their urge incontinence symptoms. The overall subjective success rate was $92 \%(n=77)$. Six women judged themselves to be improved in terms of stress incontinence but were still wearing protection, although less, to avoid any urinary leakage. One woman gave history of prickling sensation in the vagina after the procedure at her follow-up visits, from 6 weeks to 9 months although she was reassured and that prickling sensation resolved at 9 month follow-up.

Conclusion: Transobturator tape operation (outside-in technique) without cystoscopy seems an effective and safe treatment of urodynamic stress incontinence. It has a good subjective and objective outcome. A large study may be required to confirm the results.
\end{abstract}

Keywords: MONARC technique, Stress Incontinence, TOT operation.

\section{Introduction}

The rationale behind the surgical treatments for stress incontinence (SUI) in female patients has changed drastically over the last 10 years. Several techniques which are based on providing a firm platform support, but without any tension, under the midurethra have become very successful. Two techniques for TOT(Trans-obturator tape) operation have been described, the original technique as described by Delorme is based on insertion of tape through the obturator foramen from the outside-to-inside direction (skin to vaginal incision [1] and the second technique is the inside-to-outside approach described by De Leval. In this technique the tap is passed from the vaginal incision to the obturator foramen [2]

Delorme's [1] trans-obturator approach describes the objective of taking full advantage of the tensionfree sling in TVT (Tension free vaginal tape) but sparing the retropubic space. [1,3] Although long-term data 
are not available, the effectiveness and safety of this approach during the last 10 years is very promising. [4,5] Some studies have suggested that the risk of bladder injury is so low that cystoscopy needs not to be performed during this procedure [1,3, and 5]. Therefore this procedure is less time consuming and is faster to perform. The continence rate obtained with TOT has been similar to those of the retropubic TVT at least for short-term follow-up. [6,7] Initial clinical data [6,7,8] as well as studies on cadaveric dissections [3,9] showed that complication rates can be decreased significantly with the obturator approach.

Keeping in mind the lower bladder injury rates with transobturator approach, this cohort study was setup to review the objective and subjective success of this operation when performed by a single surgeon and cystoscopy was not used as a part of routine surgery.

\section{Materials and Methods}

The study population consisted of 84 consecutive women who underwent a TOT operation (MONARC) with out cystoscopy in North Cumbria NHS Trust between October 2007 and November 2008; all women presented with stress urinary incontinence alone or mixed with urge urinary incontinence or vaginal wall and uterine prolapsed were included. Exclusion criteria were pregnancy, malignancy, urinary tract infection and women not fit for surgery because of multiple health problems. The symptoms of stress urinary incontinence were assessed pre- and postoperatively by history and examination and in some cases of mixed urinary symptoms by urodynamics.

All women underwent a standardized history and complete abdominal and pelvic examination. Each woman had her own pre- and postoperative assessment data sheet, which was recorded and filed. A site-specific vaginal examination was carried out both in a supine position using Cusco's speculum and left lateral position using a Sims' speculum with maximum straining. An attending urogynaecologist performed all surgeries. Patients were followed up at 6 week and 3 month postoperatively with face to face consultation, physical examination and occasionally with urine volume measurement.

Pre-operative urodynamic evaluation was only undertaken in women with mixed urinary symptoms or symptoms of abnormal voiding.

\section{Surgical Technique}

All TOT procedures were performed with the MONARC (TOT by American medical system) subfacial hammock system (American Medical system Inc, Minnetonka, MN) using the technique recommended by Manufacturer's instruction. (Fig.1-3)

In this study, all women underwent general anaesthesia and had prophylactic antibiotics at induction. Each patient was placed in exaggerated lithotomy position with hyperflexion of the thighs that bent back on the abdominal wall. Legs were mildly abducted to expose crural area better in order to correctly place the landmarks on the skin. Examination under anaesthesia was carried out to re-evaluate vaginal support as well as any defect at all sites of the anterior, uterine, vault, and posterior vaginal wall. Patients were catheterised by using in-out urethral catheter before procedure.

The anteriolateral sulcus was anaesthetized locally by infiltrating forty millilitres of xylocaine $1 \%$ mixed with adrenaline at a concentration of 1:200,000, one side at a time. A midline vertical incision of nearly 2 $\mathrm{cm}$ in size was made in the vaginal mucosa approximately 2 centimetres below the external urethral meatus. After minimal parauretheral dissection of the vaginal wall from the urethra using a blunt scissor, the inferior pubic ramus, which forms boundary of obturator foraman was felt with the index finger. Sharp dissection was then performed to create a tunnel to the obturator foramen on either side by feeling ischiopubic ramus (a land mark used where to stop), care was taken not to injure the neurovascular bundle. Two points were then taken, first at the level where a horizontal line passing at the level of the clitoris and another vertical one delineating the outer (lateral) boundaries of the labia majora, meet, second point is taken $2.0 \mathrm{~cm}$ lateral to the first mark and at the same horizontal level represents the site of the tape exit.

A $0.5 \mathrm{~cm}$ vertical incision was made in the skin at second point bilaterally. The tip of MONARC tunneler was introduced through the skin incision downward and medially towards the obturator foramen to cross the obturator membrane. Once tunneler crossed the obturator membrane, the finger of the other hand would make contact with tunneler laterally underneath the symphysis pubis. The tunneller was then guided by the finger the vaginal incision. The tapered end of the tape was introduced into the eye of the needle and then pulled through retrieving the free end of the tape for few centimetres above the skin level. The same steps were repeated on the other side. A central mark under the urethra was made by equally pulling free ends of the tape at their skin exits. The distance between the tape and urethra was adjusted by leaving 5 millimetres gape between the two without any tension exerted by the tape on the urethra. Additional prolapse procedures were performed as required. If the patient required vaginal hysterectomy, it was performed before the tape but anterior and posterior colporrhaphy were performed after insertion of the tape. Complete haemostasis was secured, the excess edges of the tape were trimmed, with one or two stitches in the skin incision with $2 / 0$ vicryl sutures. 
Trans-Obturator Tape Operation Using Monarc Technique (Outside-In Technique) --- Revisited ..

The vaginal incision was closed with continuous $2 / 0$ vicryl sutures. Cystoscopy was not performed in patients intra-operatively unless there was suspected bladder or urethral injury. Cough test or suprapubic pressure was also not performed. The intra-operative data collected included the types of procedures performed, method of anaesthesia, and complications. The postoperative data were reviewed for the urinary retention, type of catheterization and duration of hospital stay as well as postoperative procedure-related complication. (Fig 413)

\section{Follow-up and Analysis}

Postoperative reviews were performed at 6 weeks and 3 months for objective and subjective assessment. At follow-up visit, the women have their postoperative site-specific examination as well as cough test and measurement of urine flow. Objective failure was defined as recurrence of urinary stress incontinence on cough test (Straining test). Subjectively, failure was defined as persistence of stress urinary symptoms. The follow up was taken by either a gynaecologist or a trained nurse practitioner. This ensured uniformity in the technique during follow-up visits.

Any stress urinary incontinence on stress test was recorded on the data sheet. Urinary function was also assessed subjectively through face to face questionnaire. Verbal assessment was used for sexual and bowels function. Any new symptoms were recorded.

\section{Results}

Eighty four women underwent a TOT during the study data. The mean age was 50 years (38-63). All women came with symptoms of stress urinary incontinence, mainly as sole symptom and some women had symptoms of mixed urinary incontinence. Pre-operatively, of those 84 patients, SUI was diagnosed in 62 patients while mixed urinary symptoms were present in 22 patients. Among those 22 patients urgency was the main symptom, 20 women complained of frequency, 2 had dysuria and 9 gave the history of nocturia. Other various pelvic organ prolapse symptoms were noted in the history and confirmed preoperatively on examination as well. Transobturator tape operation was carried out using MONARC subfacial hammock system in all women. Additional pelvic surgical procedures were performed according to the presence of other pelvic defects. No intraoperative complications were recorded. The estimated blood loss was $125 \mathrm{~mL}$ (range, 50-200 mL). None of the patient had suspected bladder injury intraoperatively. Transient urinary retention was observed in $8 \%(\mathrm{n}=7)$ of women in immediate postoperative period and they had delayed resumption of normal voiding within first four weeks. Three women developed urinary tract infection in immediate postoperative period and in one woman urethral pus was noted which resolved with the help of oral antibiotics. One patient was readmitted two weeks after operation with vaginal bleeding, which settled with antibiotics. That patient underwent vaginal hysterectomy in addition to tape operation and that bleeding was from the vaginal vault. Postoperative objective assessment was made with speculum examination and stress provocation tests like cough and valsalva manoeuvres. No mesh erosion was seen in any woman. There was no stress incontinence on stress provocation tests. Two women developed de novo urge incontinence that was treated with aniticholinergics. Two complained of worsening urge incontinence symptoms and none of the women having concurrent urge incontinence symptoms showed any improvement in their urge incontinence symptoms. The symptoms of urinary frequency, dysuria and nocturia improved. The overall subjective success rate was $92 \%(\mathrm{n}=77)$. Six women judged themselves to be improved in terms of stress incontinence but were still wearing protection, although less, to avoid any urinary leakage. One woman gave history of prickling sensation in the vagina after the procedure at her follow-up visits, from 3 to 9 months, she was reassured and that prickling sensation resolved at 9 month follow-up.

\section{Discussion}

During the past decade, improved understanding of the pathophysiology of female SUI has led to the development of numerous surgical techniques aimed at curing urinary incontinence. Among these, TVT and TOT have widely been used for the treatment of SUI in recent years. Since the introduction of TVT 10 years ago, it has become one of the most popular continence operations worldwide. Numerous studies have demonstrated its efficacy similar to Burch colposuspension $(10,11,12)$ However, bladder perforation is the most common complication occurring during the TVT procedure, with the incidence reported between $0.8 \%$ and $21 \%$.(13) The transobturator technique has been advocated because it avoids this retropubic passage and, at least in theory, should reduce the risk of bladder, bowel, and iliac vessel injury. Our study showed a significantly higher objective and subjective cure rate with the MONARC even when performed with out cystoscopy. The novel anatomic approach of the TOT, although avoiding the Space of Retzius and thereby reducing the risk of bladder injury, does allow for the potential for other complications including obturator neurovascular injury and lower extremity complications not seen with other approaches. Hematomas and abscesses of the obturator compartment have been reported after procedures using the transobturator approach. (14) In this study in fact intra-operatively no suspected bladder injuries occurred in all 84 patients and blood loss was minimal as well. 
Trans-Obturator Tape Operation Using Monarc Technique (Outside-In Technique) --- Revisited ...

There were no recorded bowel, blood vessel or nerve injuries. Concurrent vaginal reconstructive surgery at the time of TOT operation was not a significant independent risk factor for bladder perforation. Transient urinary retention and delayed resumption of voiding were observed in only $8 \%$ of women having surgery. This lower rate of urinary retention is consistent with a sling that is less obstructive and may be a distinct advantage of the obturator approach. The rate of lower urinary tract infection and vaginal infection was also seen in only few of the women and were cured with oral antibiotics.

Post-operatively on objective assessment with speculum examination and stress provocation tests like cough and valsalva manoeuvres there was no stress incontinence. Anticholinergics were used in 2 women who developed de novo urge incontinence. Two complained of worsening urge incontinence symptoms and none of the women having concurrent urge incontinence symptoms showed any improvement in their urge incontinence symptoms. There has been Improvement in symptoms of urinary frequency, dysuria and nocturia. This data is in consistent with previous clinical trials which suggested that complication rates can be decreased significantly with the obturator approach. Furthermore, temporary or persistent urinary retention and de-novo overactive bladder syndrome after the surgery is less with TOT because its tape is placed in a more horizontal direction than in the TVT procedure. [3, 6-9]. The overall subjective success rate was $92 \%(n=77)$. Only few of the patients were still using protection to avoid any urinary leakage.

Thus due to its location in the pelvic cavity, bladder injury could be avoided in the TOT approach. However injuries to the bladder, bladder neck, or urethra can occur if the tip of the tunneller does not follow the right course. Therefore before attempting the transobturator approach, it is necessary to become familiar with the surgical anatomy involved in the procedure. Whiteside and Walters [3] described the anatomic study on six cadavers to determine the relationships between a transobturator tape and the anatomic structures within the obturator region. The TOT device in the outside-to-inside approach passed through, in order, gracilis, adductor brevis, obturator externus, obturator membrane, beneath or through obturator internus, periurethral endopelvic connective tissue, and finally came out to the vaginal incision. Using the index finger to reach the tip of the tunneller and guide it through the dissected path appears to be a crucial part of the procedure in order to avoid injuring those structures. The TOT passes below the levator ani and did not enter the space of Retzius, thus reducing the risk of bladder injury significantly.

This study is limited by small number of women and its retrospective nature. The study's retrospective design precludes definite conclusions about the cause and effect of observed differences. These findings should be further evaluated with randomised, controlled trials.

Despite these limitations transobturator tape operation (outside-in technique) without cystoscopy seems an effective and safe treatment of urodynamic stress incontinence. It has a good subjective and objective outcome.

\section{Acknowledgements}

The author would like to thank Mr Mohammad Matar and American Medical Systems whose involvement made this study possible.

There is no conflict of interest.

\section{Conflict of interest}

\section{References}

[1]. Delorme E (2001) Transobturator urethral suspension: mini-invasive procedure in the treatment of stress urinary incontinence in women. Prog Urol; 11:1306-1313

[2]. De Leval J (2003) Novel surgical technique for the treatment of female stress urinary incontinence: transobturator vaginal tape inside-out. Eur Urol; 44:724-730.

[3]. Whiteside JL, Walters MD (2004) Anatomy of the obturator region: relations to a trans-obturator sling. Int Urogynecol J Pelvic Floor Dysfunct; 15:223-226.

[4]. David-Montefiore E, Frobert JL, Grisard-Anaf M, Lienhart J, Bonnet K, Poncelet C, et al (2006) Peri-operative complications and pain after the suburethral sling procedure for urinary stress incontinence: a French prospective randomised multicentre study comparing the retropubic and transobturator routes. Eur Urol;49:133-138

[5]. Naidu A, Lim YN, Barry C, Goodwin S, Corstiaans A, Rane A (2005) Transobturator tape for stress incontinence: the North Queensland experience. Aust N Z J Obstet Gynaecol;45:446-449.

[6]. 6.deTayrac R, Deffieux X, Droupy S, et al (2004) A prospective randomized trial comparing tension-free vaginal tape and transobturator suburethral tape for surgical treatment of stress urinary incontinence. Am J Obstet Gynecol; 190:602-608

[7]. Mellier G, Benayed B, Bretones S, et al (2004) Suburethral tape via the obturator route: is the TOT a simplification of the TVT? Int Urogynecol J Pelvic Floor Dysfunct; 15:227-232

[8]. Costa P, Delmas V (2004) Trans-obturator-tape procedure: "inside out or outside in": current concepts and evidence base. Curr Opin Urol; 14:313-315.

[9]. Bonnet P, Waltregny D, Reul O, et al (2005) Transobturator vaginal tape inside out for the surgical treatment of female stress urinary incontinence: anatomical considerations. J Urol; 173:1223-1228.

[10]. 10.Paraiso MF, Walters MD, Karram MM, Barber MD (2004) Laparoscopic Burch colposuspension versus tension-free vaginal tape: a randomized trial. Obstet Gynecol;104:1249-1258 
Trans-Obturator Tape Operation Using Monarc Technique (Outside-In Technique) --- Revisited ..

[11]. 11. Ward KL, Hilton P (2004) A prospective multicenter randomized trial of tension-free vaginal tape and colposuspension for primary urodynamic stress incontinence: two-year follow-up. Am J Obstet Gynecol;190:324-331.

[12]. 12. Meschia M, Pifarotti P, Bernasconi F, Guercio E, Maffiolini M, Magatti F, et al (2001) Tension-free vaginal tape: analysis of outcomes and complications in 404 stress incontinent women. Int Urogynecol J Pelvic Floor Dysfunct;12(Suppl 2):S24-S27.

[13]. 13. Atherton MJ, Stanton SL (2005) The tension-free vaginal tape reviewed: an evidence-based review from inception to current status. BJOG; 112:534-546.

[14]. Goldman HB (2006) Large thigh abscess after placement of synthetic transobturator sling. Int Urogynecol J Pelvic Floor Dysfunct; 17:295-296.

\section{Diagrams $(1-13)$}

Fig 1. Schematic Diagram of TOT

Fig 2. MONARC Curved

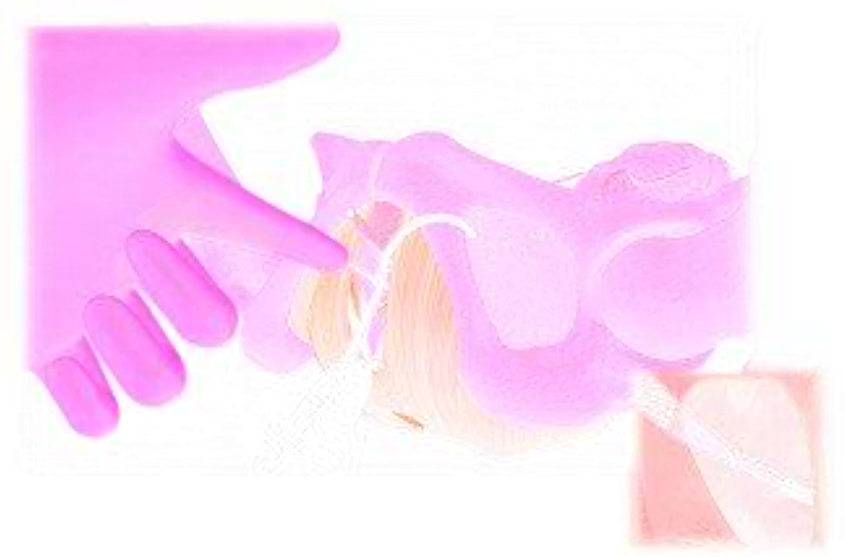

Fig 3. Manufacturer's Instructions on use of MONARC.

Fig 4. Vaginal Incision. 


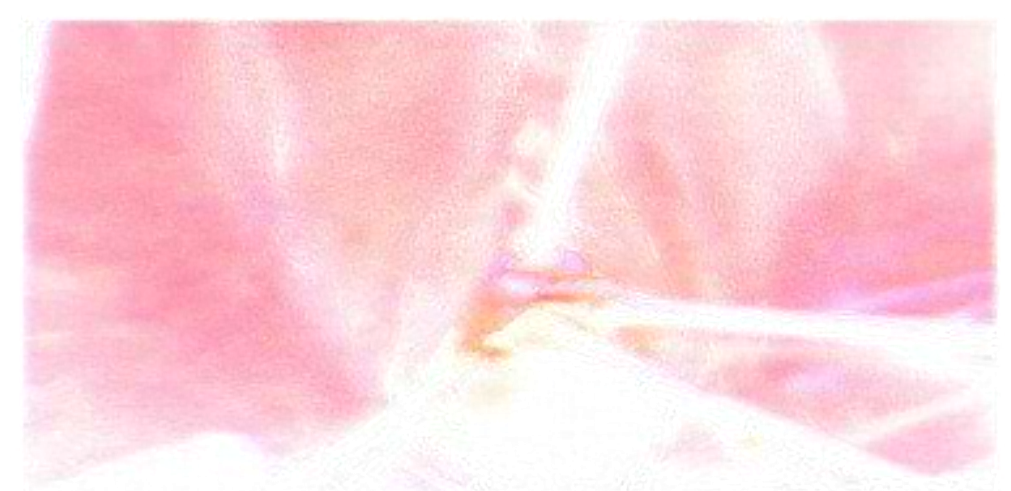

Fig 5. Sharp dissection

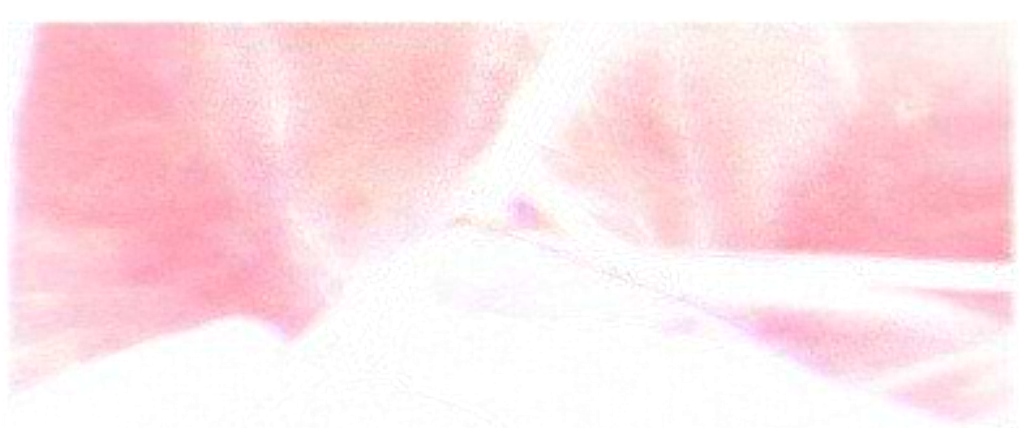

Fig. 6. Blunt Dissection

Fig 7. Evaluating Obturator foramen

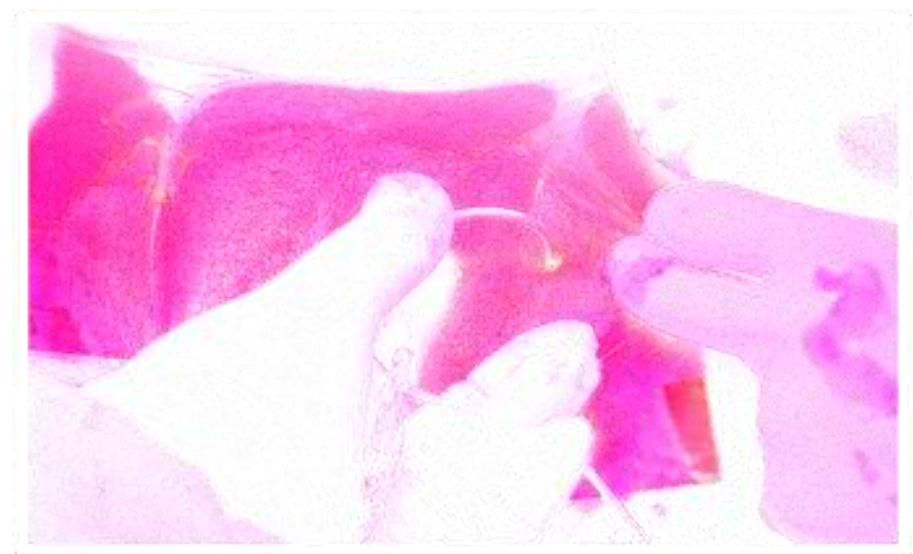

Fig 8. Introducing MONARC curved 


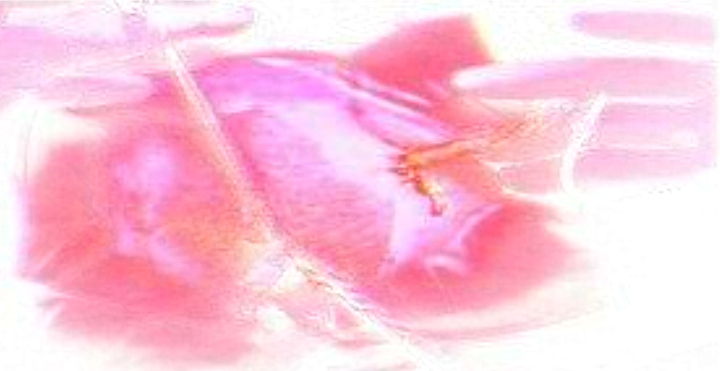

Fig 09. Both ends of the tape identified

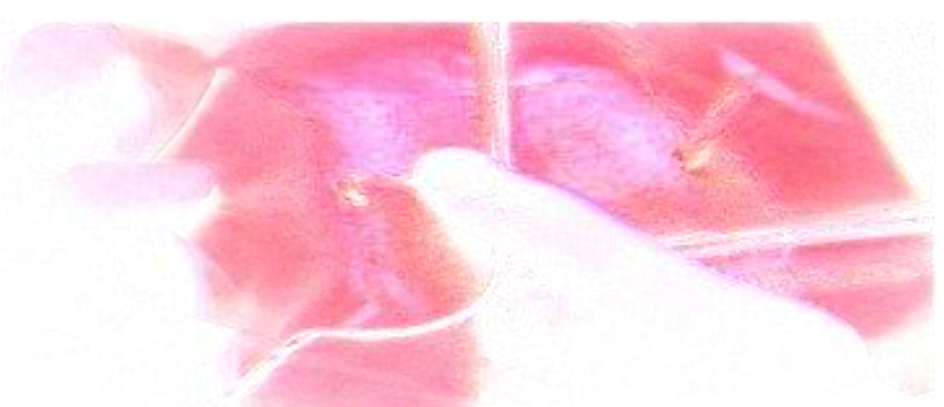

Fig 10. Similar TOT procedure on other side

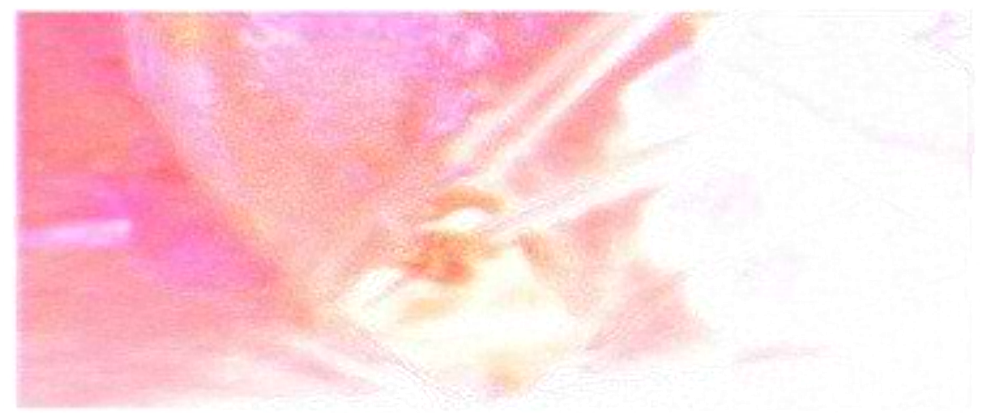

Fig 11. Tension adjusted

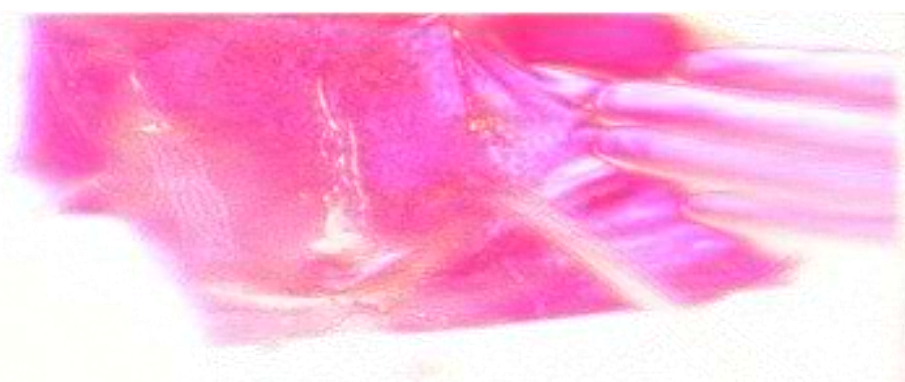

Fig 12. TOT ends trimmed

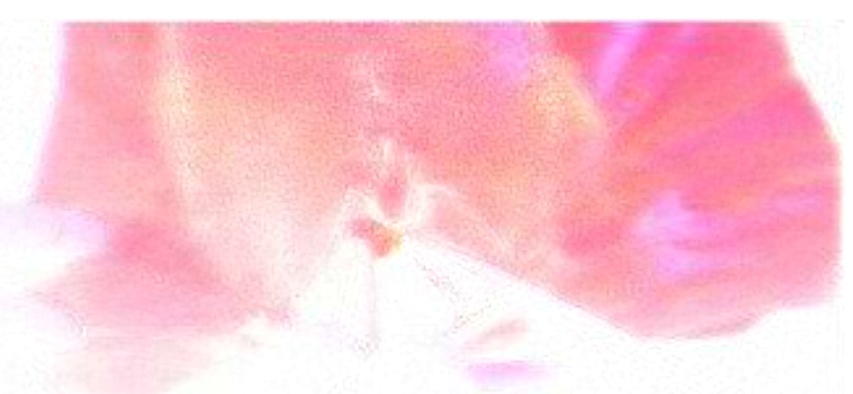

Fig 13. Vaginal wall sutured 


\section{Diagrams}

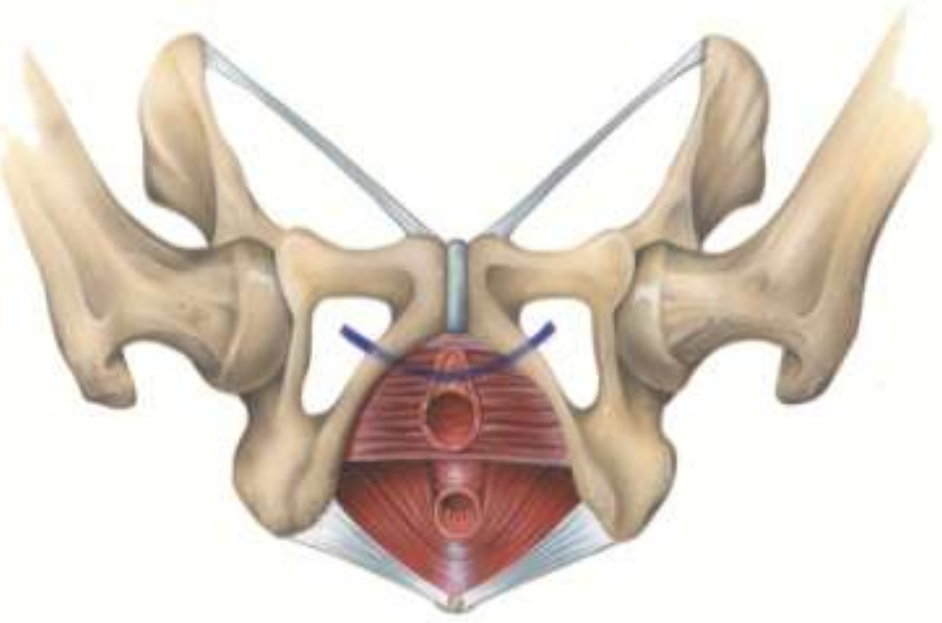

Fig 1. Schematic Diagram of TOT

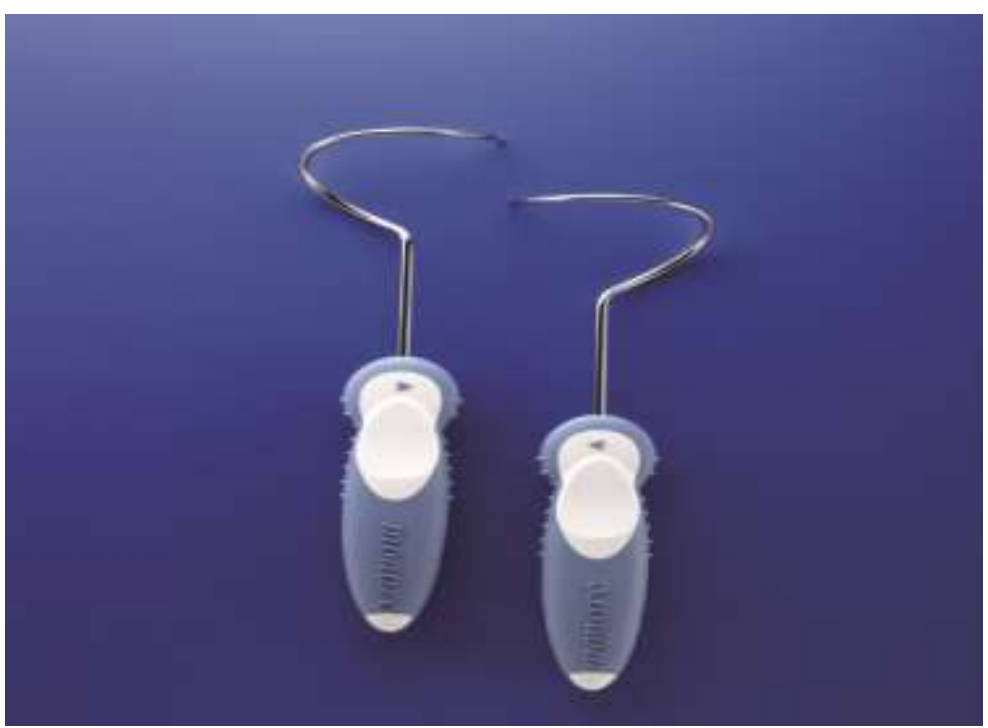

Fig 2. MONARC Curved

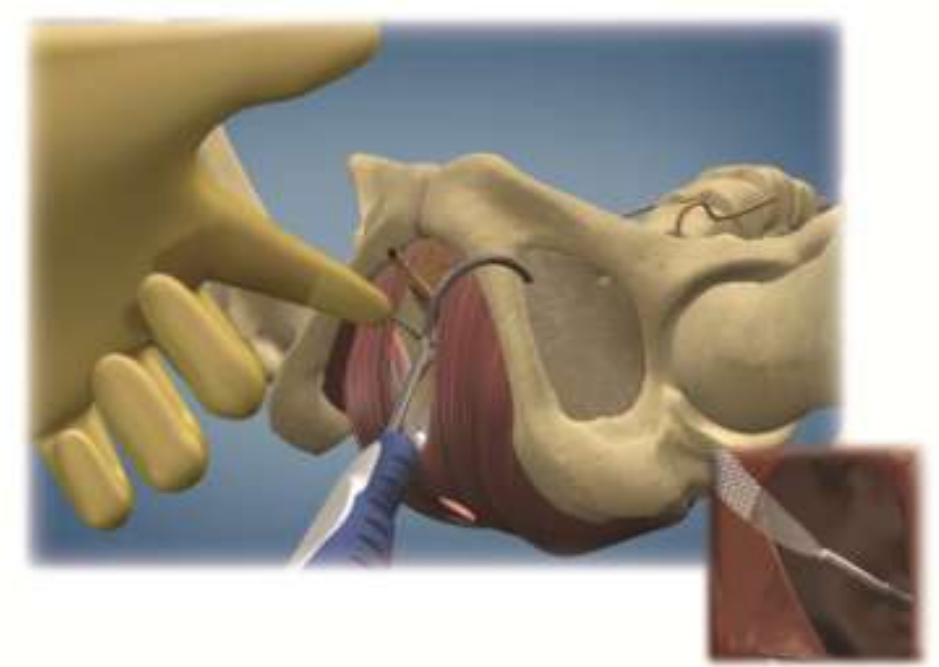

Fig 3. Manufacturer's Instructions on use of MONARC. 


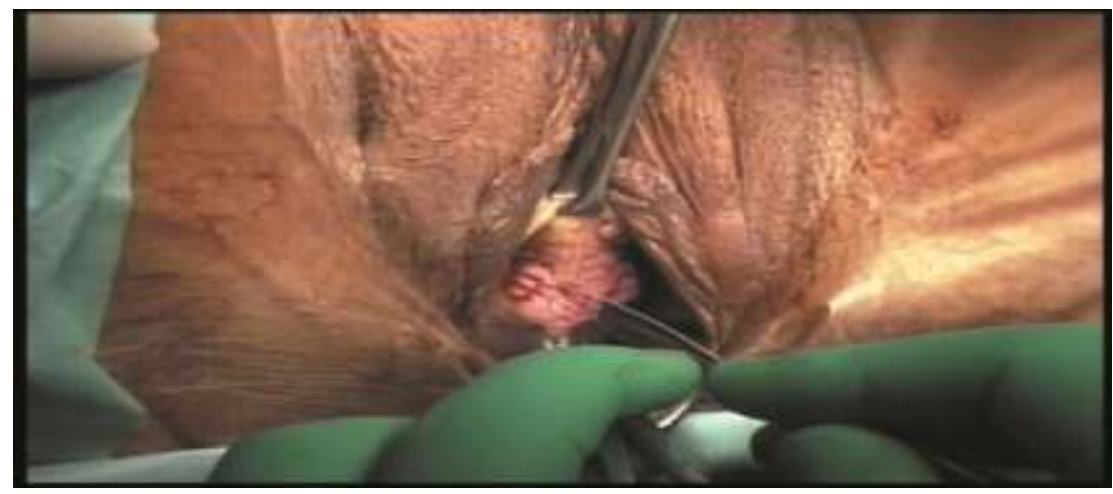

Fig 4. Vaginal Incision.

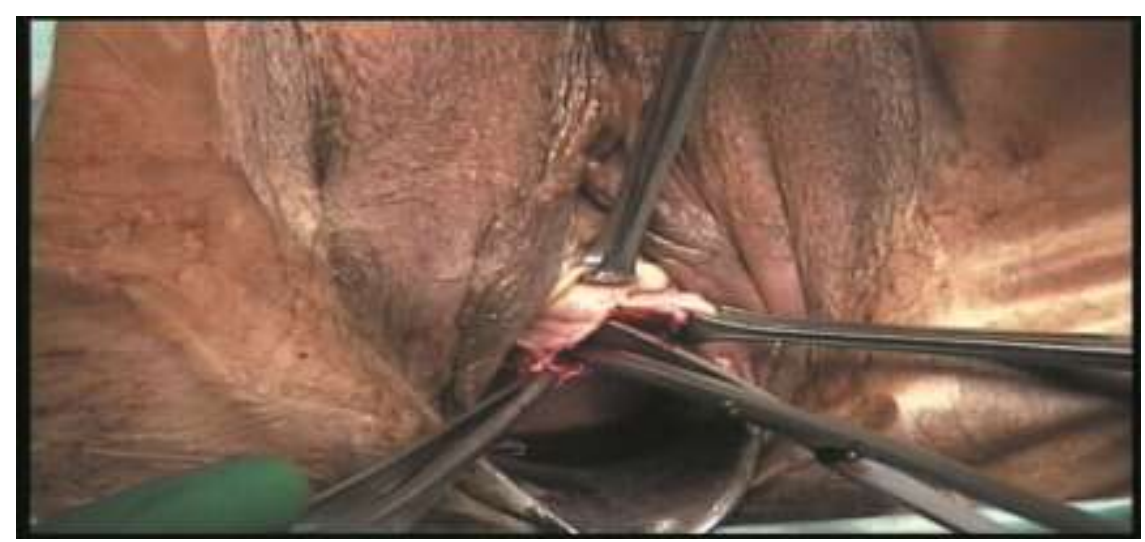

Fig 5. Sharp dissection

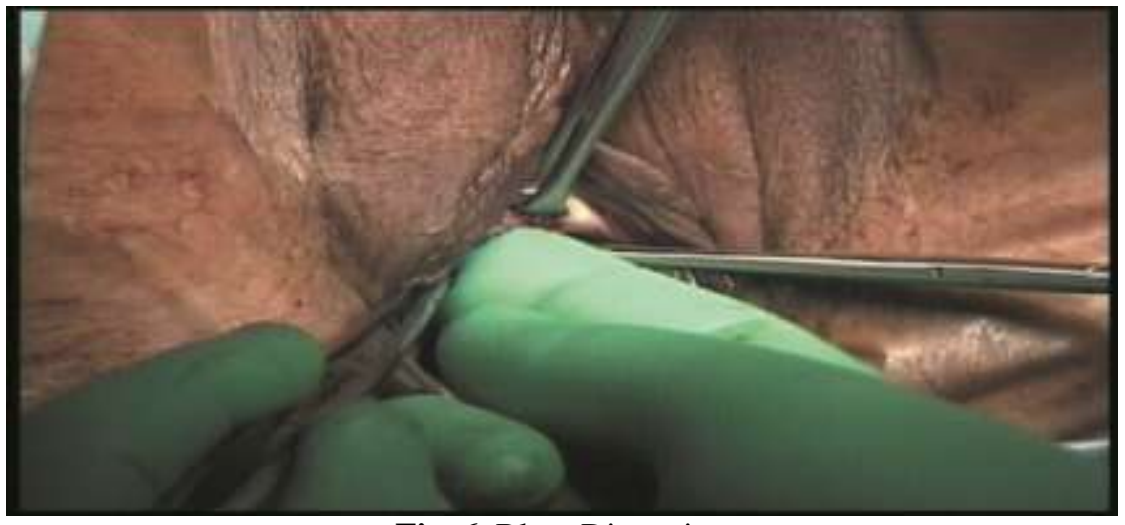

Fig. 6. Blunt Dissection

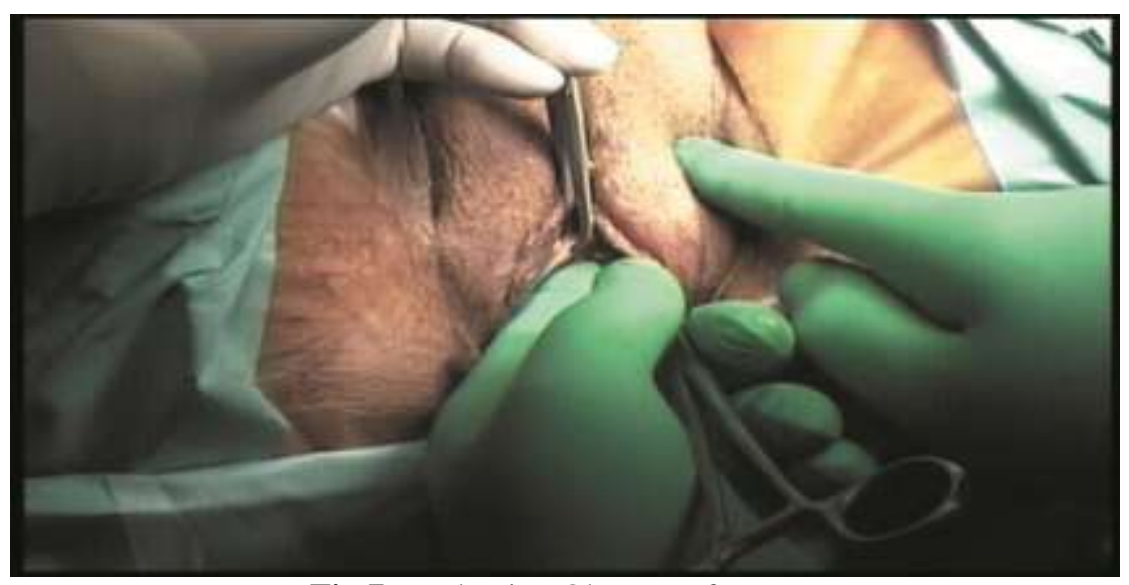

Fig 7. Evaluating Obturator foramen 


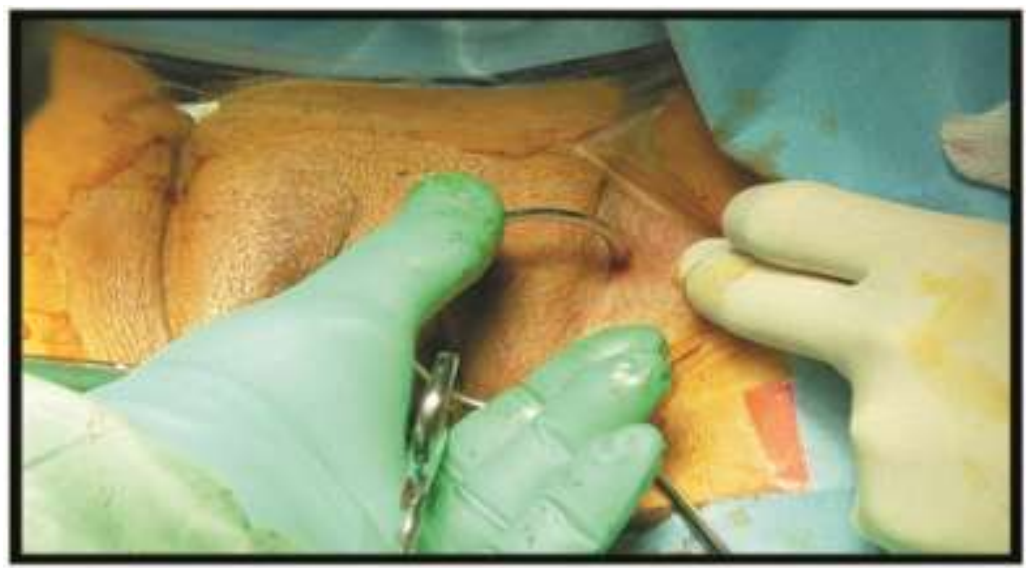

Fig 8. Introducing MONARC curved

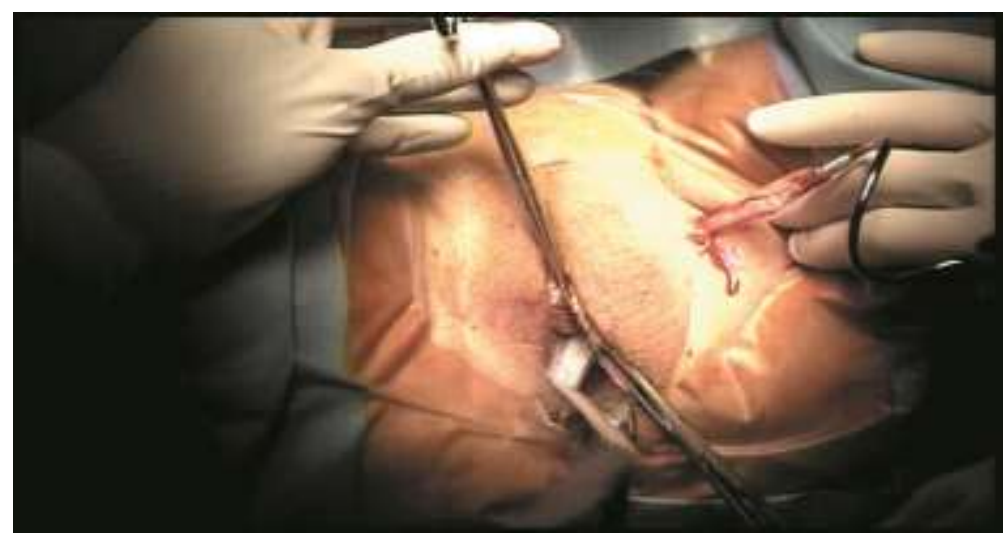

Fig 09. Both ends of the tape identified

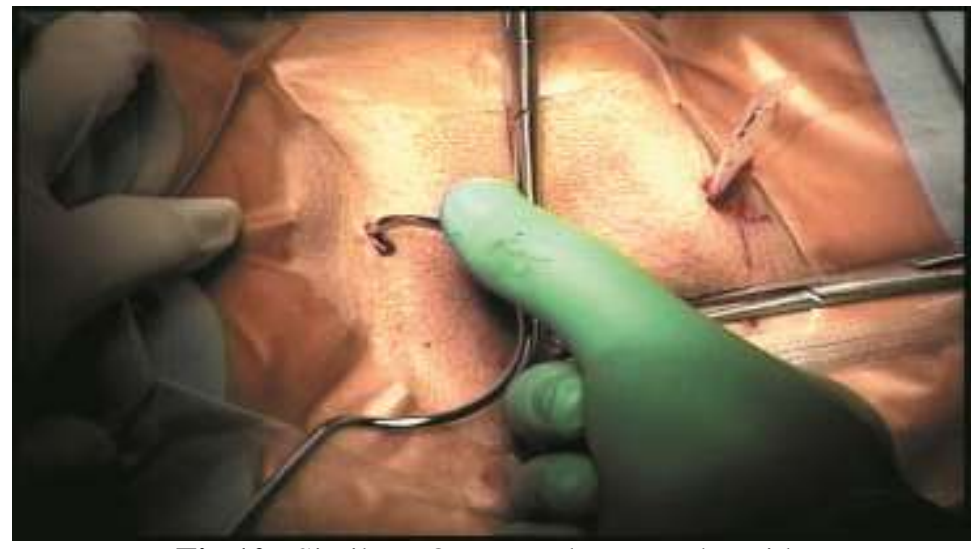

Fig 10. Similar TOT procedure on other side

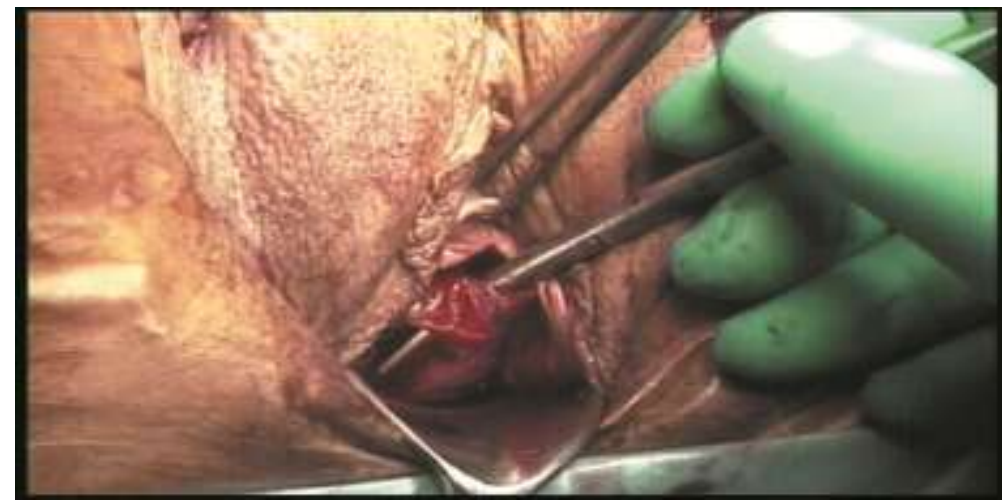

Fig 11. Tension adjusted 


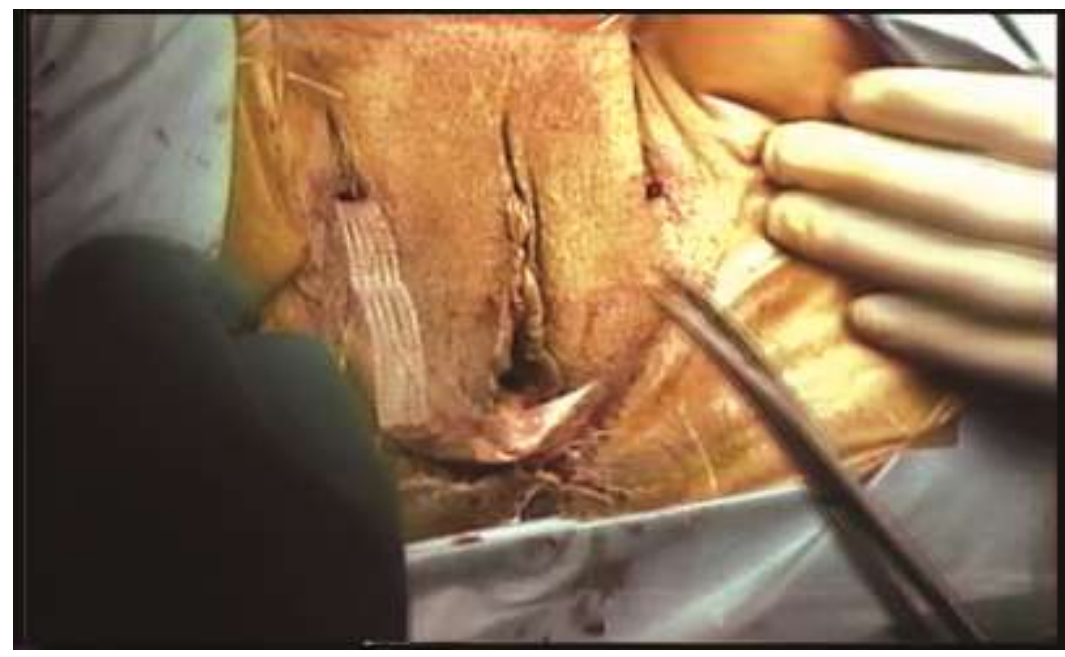

Fig 12. TOT ends trimmed

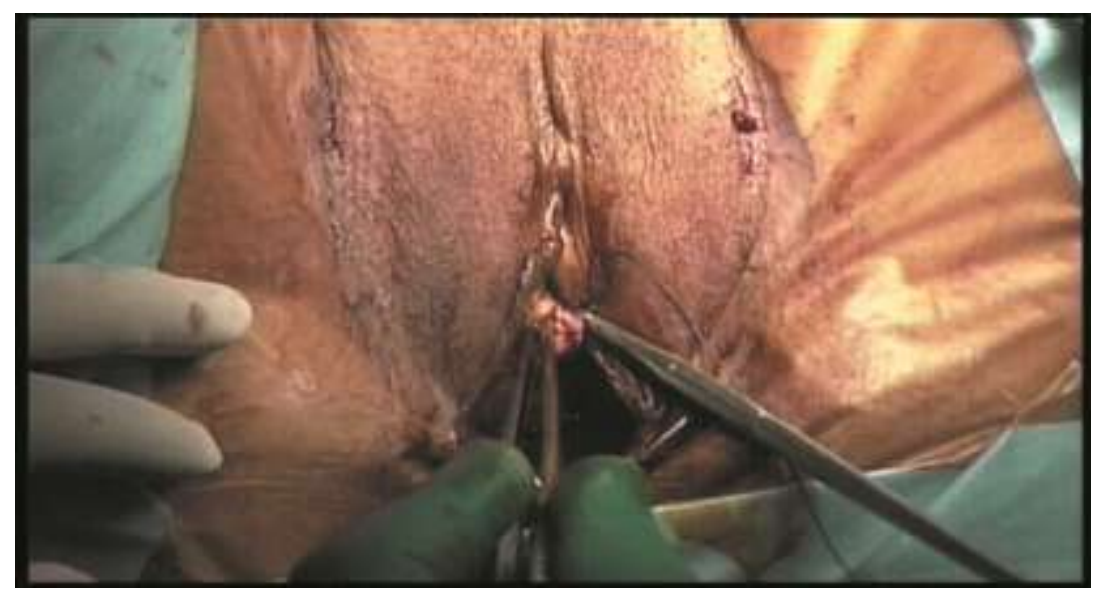

Fig 13. Vaginal wall sutured 